\title{
Retrograde Single Elastic Intramedullary Nail in Closed Simple Diaphyseal Humeral Shaft Fractures in Children
}

\author{
Mohamed M H El-Sayed MD, Ahmed F. Shams MD², Osama Gamal ${ }^{3}$, \\ Mohamed EISawy ${ }^{4}$ \\ ${ }^{1}$ Department of Orthopedics \& Traumatology, Tanta University \\ ${ }^{2,3 \& 4}$ Department of Orthopedics \& Traumatology, Menofeya University. Egypt \\ mhosney2012@hotmail.com
}

\begin{abstract}
Humeral shaft fractures in children are very infrequent and only represent 2-5\% of all pediatric fractures. They are predominantly seen in children aged less than 3 years or more than 12 years. Fractures of humeral shaft may result due to direct or indirect forces. The most frequent site of the fracture is between the middle and the distal thirds of humerus. Several series of antegrade intramedullary stabilization of humeral fractures (Rush rods and Ender nails) have been reported. In the literature, several complications such as shoulder impingement and adhesive capsulitis have been reported with this technique. In this study, the functional and radiological results of management of closed fractures of the humerus in children using a single retrograde elastic intramedullary nail shall be evaluated.
\end{abstract}

Keywords: Level of Evidence: Case series, level IV

\section{INTRODUCTION}

Fractures of the humeral shaft usually result from a direct force such as; a direct impact, road traffic accidents and/or crush injuries. Indirect forces such as; a fall on the elbow, or an out stretched hand, or even strong muscular contractions can cause these fractures. The most frequent site of the fracture is between the middle and the distal thirds of humerus. ${ }^{[1,2]}$

The simplest classification of humeral shaft fractures is based on the location of the fracture site in the humeral diaphysis (proximal, middle, and distal), alignment of the fracture fragments, and the appearance of the fracture line ${ }^{[3]}$ Radial nerve injury is the most common associated injury due to the close proximity of this nerve to the bone, especially in fractures of the middle third. ${ }^{[4,5]}$

Functional bracing that is frequently indicated for stable fractures with adequate alignment can most often treat diaphyseal fractures of the humerus in children non-operatively. Noncompliance, in addition to limited fracture stabilization, are the main complications encountered with this type of treatment. ${ }^{[2]}$ Children with humeral shaft fractures require operative treatment only frequently, primarily in cases where surgical stabilization of humeral shaft fractured fragments is required, to assist patient mobilization, in poly-trauma patients, for wound care in open fractures, to maintain adequate alignment, and/or in cases with failed conservative measures. ${ }^{[6][7][8]}$

Although rigid plate osteosynthesis is the most widely accepted operative method, it carries many disadvantages including; extensive soft tissue surgical trauma, loss of the fracture hematoma, significant blood loss, increased operative time, prolonged hospitalization period, delayed rehabilitation, and the risk of intra-operative radial nerve injury. In addition, all the patients are subjected to another surgery for plate removal. ${ }^{[9]}$

Several series of antegrade intramedullary stabilization of humeral shaft fractures (Rush rods and Ender nails) have been reported in the literature. Problems of shoulder impingement and adhesive capsulitis of the shoulder were a significant problem in these series, because most of the nails were inserted in an antegrade fashion through a small incision in the rotator cuff. ${ }^{[7][10]}$

Since publication of outcomes of Spanish and Nancy groups in the early 1980s, elastic stable intramedullary nailing (ESIN) has become a well-accepted method of surgical treatment of diaphyseal fractures of long bones in children and adolescents. ${ }^{[4]}$

In the literature, all the published series were performed using 2 elastic intramedullary nails for fixation of diaphyseal fractures of the humerus in children. In most of the studies the nails were 
inserted in an antegrade manner, while in very few cases, a retrograde insertion was performed. Nevertheless, in all the published reports 2 nails were inserted. A question was raised, would a single retrograde intramedullary elastic nail be sufficient for stabilization of closed simple diaphyseal fractures of the humerus in children? In this study the final functional and radiological results of management of such fractures in children using a single elastic intramedullary nail inserted in a retrograde fashion shall be presented.

\section{Patients And Methods}

Between January 2011 and January 2012, all the patients suffering from simple closed fractures of the humeral shaft were included in this study. There were 23 patients. Of the studied patients there were 9 cases with a transverse fracture line, (<30 degrees), (AO fracture type 12- A3), 8 cases with an oblique fracture line, ( $>30$ degrees), (AO fracture type 12- A2), while the remaining 6 cases presented with a spiral fracture line, (AO fracture type 12- A1) (Figure 1 ).

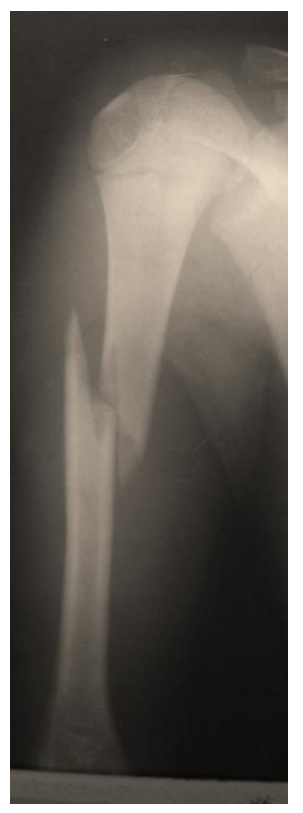

Figure1. Pre-Operative X-Ray of a Male Patient, 8 Years Old, with a Closed Right-Sided Spiral Fracture of the Middle Third of the Humerus

All the studied cases were treated at an academically supervised Pediatric Trauma Center. After parental written consent, all the patients were treated using the same technique of retrograde single elastic intramedullary nail. There were 19 males and 4 females, 15 with right arm fractures and 8 with left arm fractures. The mean age was 10 years old at the time of surgery, with a range from 7 to 12 years. The initial clinical and radiological examination revealed that 9 patients had transverse fractures while 14 patients had an oblique or spiral fracture line. Radial nerve palsy was recorded in 2 cases before the index surgery. One patient had an ipsilateral injury of the lower radial epiphysis Salter Harris type II, that was managed concomitantly using closed reduction under radiographic guidance and Kirschner wire fixation. Patients with pathological fractures, and/or open fractures were excluded from this study.

The time lapse between trauma to surgery ranged from 3 hours to 36 hours, after referral, with a mean of 8 hours. A single prophylactic antibiotic was administered 2 hours before the index surgery in the form of a third generation cephalosporin injection, and the dose was adjusted according to the age and weight of the patient. General anaesthesia was given to all the affected individuals in the supine position. An orthopedic radiolucent table was used in all the cases to allow for the use of radiographic control (C-arm).

A 5-10 millimeter, (mm), skin incision was made over the lateral distal end of the humerus just overlying the lateral supracondylar region just above the level of the lateral humeral epicondyle. The point of insertion was about $2 \mathrm{~cm}$ above the growth plate. (Figure 2) An oblique hole was done with a drill bit or an awl at least $0.5 \mathrm{~mm}$ greater than the diameter of the planned flexible nail. Proper selection of the nail diameter was performed based on the width of the affected intramedullary canal. The rule was never to exceed $40 \%$ of the diameter of the narrowest part of the medullary canal. Nail 
contouring into a $\mathrm{C}$ shaped configuration, with the tip of the curve at the apex of the fracture site. The arch of the curve of the nail should be three times the diameter of the bone into which it shall be inserted. (Figure 3)

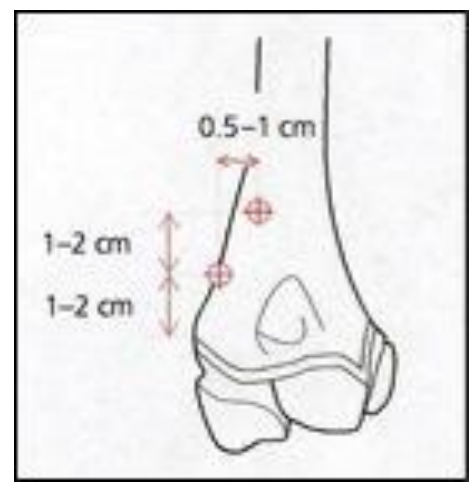

Figure2. Point of Entry of the Flexible Intramedullary Nail

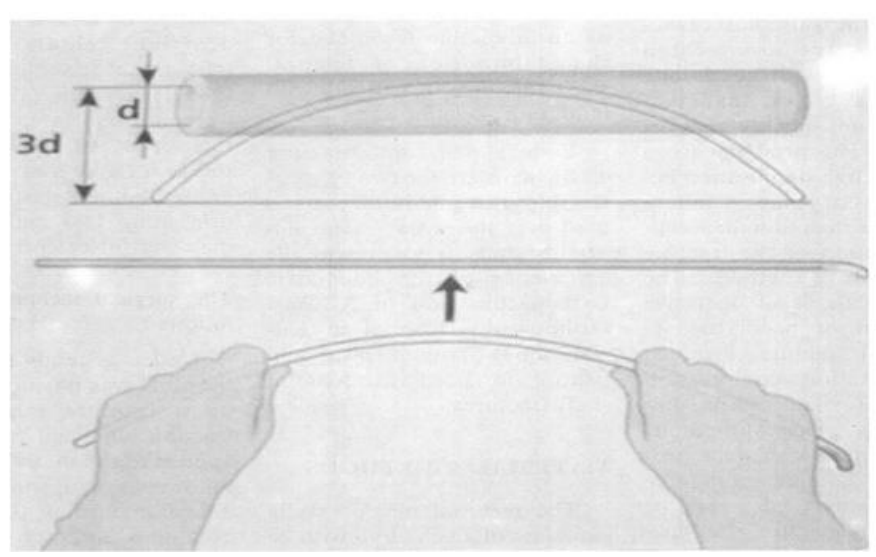

Figure3. Contouring of the Flexible Intramedullary Nail before Insertion

The flexible nails were bent in order to achieve a three-point fixation at the fracture site. Retrograde insertion of the properly selected nail was then performed. Once the nail reached the fracture site, closed reduction was performed under $\mathrm{C}$-arm control. After proper alignment and fracture reduction, the nail was introduced into the proximal fragment. Multiple and forceful reduction attempts were avoided. Curvature of the nail facilitated some reduction and could be rotated to achieve the best alignment. The nail was driven proximally to within $1-2 \mathrm{~cm}$ from the proximal humeral physis. The protruding distal end of the nail was then trimmed at $0.5 \mathrm{~cm}$ from the bone for easy removal later on. The single nail acted as an internal splint and maintained the alignment until the fracture has healed. Technical details were important and carefully performed in placing the nails in the retrograde fashion.

The patients were then instructed to keep their arms in a sling or a functional brace for not more than 3 weeks. (Figure 4) During this period, the patients were advised to remove the sling two to five times daily and perform pendulum exercises. From the fourth week onwards, they were encouraged to progress from passive assisted to active exercises as tolerated.
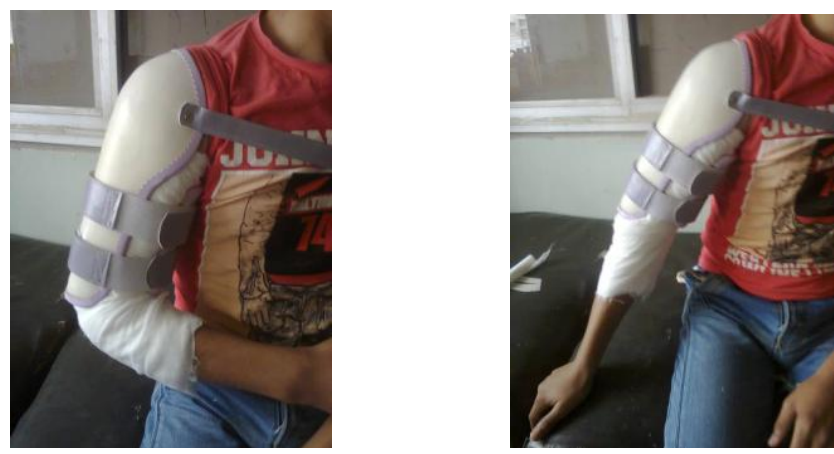

Figure4. Functional Bracing was encouraged for About three weeks 
Periodic regular clinical and radiographic evaluation was performed for all the studied cases. (Figure 5) The functional outcome was evaluated according to the system of Broberg and Morrey $(11,12)$, the DASH score (13), and was compared to the healthy side. The Broberg and Morrey system is a primary short-term outcome measure. This 100-point rating system is based on motion (40 points), strength (20 points), stability ( 5 points), and pain (35 points). The surgeon rates pain as none (35 points), mild with activity but requiring no medication ( 28 points), moderate with or after activity (15 points), or disabling pain that is severe at rest and requires constant medication ( 0 points). Categorical ratings are assigned according to the score achieved: a score of 95 to 100 points is rated excellent; 80 to 94 points, good; 60 to 79 points, fair; and <60 points, poor. Solid union was confirmed when at least three cortices showed periosteal new bone formation on standard plain antero-posterior and lateral views, and radiological follow-up was graded according to the system proposed by Anthony et al (Grading of Callus Formation) (14). Although this system was at first designed for the follow-up of the diaphyseal femoral fractures, it was found useful and easily applicable for all diaphyseal fractures of long bones. (Table 1)
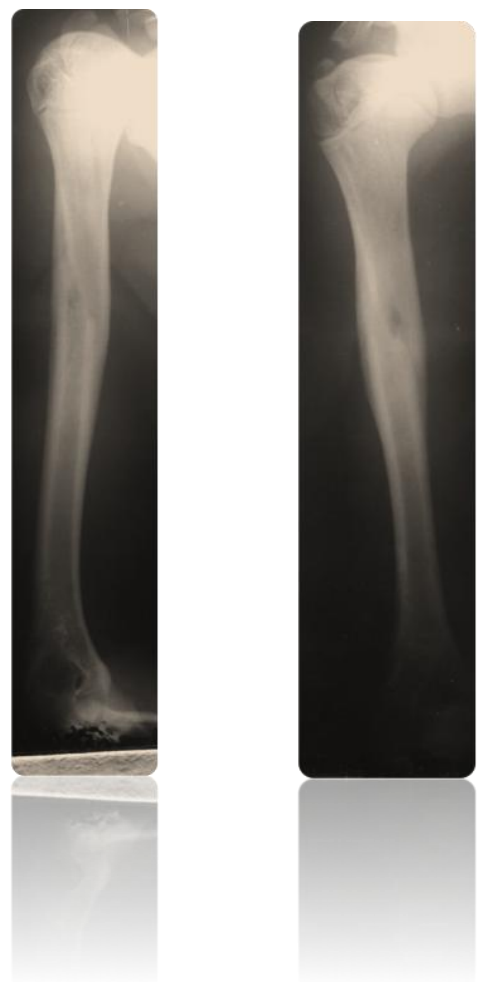

Figure5. Immediate Post-Operative Antero-Posterior and Lateral Radiograph Performed for the Same Patient. And the Final X-Rays taken after Removal of the Nail and Solid Consolidation

\begin{tabular}{|c|c|}
\hline Grade 0 & No identifiable fracture healing \\
\hline Grade 1 & Primary bone healing with little or no periosteal new bone formation \\
\hline Grade 2 & Periosteal new bone formation on two sides of the femur \\
\hline Grade 3 & Periosteal new bone formation on three or four sides of the femur \\
\hline
\end{tabular}

Table1. Grading of Callus Formation, and Bone Healing

\section{RESUltS}

For all the included patients the following data was documented: time lapse from injury to surgery, operative time, hospital stay, time to bone healing, time of removal of the implant, range of motion at the shoulder and elbow joints, functional status, humeral length discrepancy, if any, angulation and /or rotation at the fracture site, if any, and complications, if any. The humeral length discrepancy was measured clinically by comparing the distance from the medial epicondyle to the coracoid, and was compared to the healthy side. The duration from trauma to surgery ranged from 3 hours to 36 hours, with a mean of 8 hours. The mean operative time was 30 minutes (range, 20-45 min.), and the hospital stay 1.3 days (range, $1-3$ days). 
Solid union took place in all the studied cases, and was assured both clinically and radiographically within a mean of 8 weeks, (range from 5-10 weeks). There was no blood loss recorded with this technique. Implant removal was performed after a mean of 5.8 month (range, 4-7 month). Active exercises were allowed according to patients' tolerance. At the end of the minimum follow-up period of 12 months, all the patients were examined both clinically and radiographically and the functional scoring systems were completed. According to the Broberg and Morrey system; there were 20 excellent results (95 to 100 points), (86.9\%), while in only 3 cases, (13.1\%), the results were graded as good (80 to 94 points). Nevertheless, at the time of the final follow-up examination, all the studied cases showed excellent results (95 to 100 points), both clinically and radiographically. All the X-rays at the final follow-up visit were graded as Grade III. In the current study the complications encountered were; superficial wound infection in 6 cases, and nail migration occurred in 3 cases. Both recorded complications were managed adequately and did not affect the final clinical or radiographic end results. Complications such as: deep infection, growth plate injury, neuro-vascular injury, refracture, implant failure, limb length discrepancy, delayed union, non-union, and/or mal-alignment, were not recorded in this study. The two patients with evident preoperative radial nerve palsy, improved spontaneously after surgery and there was no need for intervention, and this associated injury did not affect the final clinical or radiologic outcome of the affected patients.

\section{Discussion}

The forgiving physiology of children will ensure healing, their impressive remodeling potential will at least partially compensate the deformity, and the great mobility of the shoulder joint will make any restriction of movement look trivial. ${ }^{[1][15]}$

Probably the most significant advantage of ESIN is the avoidance of lengthy and awkward immobilization regimes without compromising the reduction. Early post-operative mobilization is the rule, and children can go back to school once they are discharged from hospital. In exchange the child has to undergo two open procedures under general anesthesia. Not surprisingly, this type of treatment seems to have become a remote choice for the modern orthopedic surgeon. ${ }^{[1][2][15]}$ It will be very difficult indeed to convince a teenager to wear a brace or a cast for a long period, especially at school or in the hot summer months. The advantages of the method outweigh the risks so clearly, that it has become the recommended method of treatment for severely displaced fractures of the mid shaft humerus in children. ${ }^{[3][10][16][17]}$

Titanium elastic nail fixation is an ideal procedure for treating humeral shaft fractures in which stabilization is indicated as it provides stable fixation, with minimal soft tissue stripping at the fracture site, and allows early mobilization of the extremity. Surgical stabilization of humeral shaft fractures in children, when indicated, can be safely performed using titanium elastic nails. ${ }^{[6]}$

In the current study, the mean follow up period was 23 month with a range from 12 to 34 month. The mean time for fracture union was 8 weeks, and ranged from 5-10 weeks. The average delay from injury to surgery was from 3 hours to 36 hours, with a mean of 8 hours. The mean operative time was 30 min. (range, 20-45). Whereas radiological healing, (Grade III Anthony et al classification), was evident at 10 weeks (range, 8-14). Implant removal took place at an average of 5.8 months (range, 47).

In this study, two patients had a preoperative radial nerve injury, (neuropraxia), that recovered spontaneously. Grag in his study had one patient with a radial neuropraxia that recovered function spontaneously within 4 months after injury and the other patient with radial nerve was treated with tendon transfers approximately 1 year after the injury to restore wrist extension. ${ }^{[6]}$

Gordon in his study, all fractures went on to union in acceptable alignment. There have been no delayed unions, refractures, or malunions. There have been no postoperative wound infections. Nail migration within the first 6 weeks postoperatively has been the most frequent complication. ${ }^{[2]}$ In this study, five patients had a superficial wound infection and only 3 had nail migration.

Lascombes used two nails introduced through the distal lateral edge of the humerus above the lateral epicondyle. The first nail has a convexity toward the lateral cortex, and the second nail is rotated 180 degrees to be medially oriented. Stability is enough to lead to healing, with a very low rate of complication. ${ }^{[10]}$ In this study, the use of single pre-bent nail achieved good alignment and reduction and acted as an internal splint till fracture healing took place and this decreased the operative time and 
cost with a final favorable end result and the same low complication rate. The nail maintained the reduction and then prevented postoperative displacement, mal-alignment, and rotational displacement because it was contoured into a "C" shape construction, with the apex of the curve at the fracture site to allow for a three point fixation and a stable reduction of the fracture site. ${ }^{[18]}$

Furlan in his study, the Length of hospital stays varied between 2-3 days. All patients achieved complete radiographic healing at a mean of 7.2 weeks (range 5-11 weeks). ${ }^{[1]}$ In this study, the mean hospital stays was 1.3 days (range, 1-3). The mean time to clinical healing was 8 weeks range from 510 weeks. Whereas radiological healing was evident at 10 weeks (range 8-14)

For the past 30 years, the ESIN method has become widely accepted, and is considered the standard surgical procedure for treatment of diaphyseal fractures in children and adolescents. It has many advantages over the use of plates and external fixators, which have few indications for use in this age group. A good knowledge of the principles and strict careful application of the techniques is mandatory to obtain excellent results. This method is reliable and children benefit from its use. ${ }^{[1][15]}$

To conclude, the use of a single intramedullary elastic retrograde nail for fixation of the simple closed diaphyseal fractures of the humerus proved to be an adequate fixation technique with a very low complication rate and a favorable final clinical outcome after a short period of follow-up. When using a single nail instead of two nails; the operative time was decreased, less radiological exposure was ensured, the cost was lowered, and nevertheless, the final outcome was not compromised. In addition, there was no need for a second entry from the medial or the lateral side. Furthermore, no nail twisting or winding over each other took place when using a single flexible nail.

There were no conflicts of interest for this article, and none of the authors has received any financial support from any governmental or private association.

\section{REFERENCES}

[1] Furlan D, Pogorelić Z, Biočić M, Jurić I, Budimir D, Todorić J, Et Al. Elastic Stable Intramedullary Nailing For Pediatric Long Bone Fractures: Experience With 175 Fractures. Scand J Surg 2011;100(3):208-15.

[2] Gordon JE, Garg S. Pediatric Humerus Fractures: Indications And Technique For Flexible Titanium Intramedullary Nailing. J Pediatr Orthop 2010;30(2):S73-S76.

[3] Shaw BA, Murphy KM, Shaw A, Oppenheim WL MM. Humerus Shaft Fractures In Young Children: Accident Or Abuse? J Pediatr Orthop 1997;17:293-7.

[4] Journeau P. Flexible Intramedullary Nailing In Children. The Nancy University Manual. In: Lascombes P, Editor. Berlin Heidelberg: Springer-Verlag; 2010. Page 99-114.

[5] Ekholm R, Ponzer S, Törnkvist H, Adami J, Tidermark J. The Holstein-Lewis Humeral Shaft Fracture: Aspects of Radial Nerve Injury, Primary Treatment, and Outcome. J Orthop Trauma 2008; 22(10):693-7.

[6] Garg S, Dobbs MB, Schoenecker PL, Luhmann SJ, Gordon JE. Surgical Treatment of Traumatic Pediatric Humeral Diaphyseal Fractures with Titanium Elastic Nails. J Child Orthop 2009; 3(2):121-7.

[7] Musgrave DS, Mendelson SA. Pediatric Orthopedic Trauma: Principles In Management. Crit Care Med 2002; 30(11):431-43.

[8] Lavelle WF, Uhl R, Krieves M, Drvaric DM. Management Of Open Fractures In Pediatric Patients: Current Teaching In Accreditation Council For Graduate Medical Education (ACGME) Accredited Residency Programs. J Pediatr Orthop B 2008;17(1):1-6.

[9] County H. Operative Treatment Of Humeral Shaft Fractures: Plates Versus Nails. Tech Shoulder Elb Surg 2001; 2(3):194-209.

[10] Lascombes P, Haumont T, Journeau P. Use and Abuse Of Flexible Intramedullary Nailing In Children And Adolescents. J Pediatr Orthop 2006; 26(6):827-34.

[11] Broberg MA, Morrey BF. Results Of Delayed Excision Of The Radial Head After Fracture. J

[12] Bone Joint Surg Am. 1986 Jun; 68(5):669-74.

[13] Broberg MA, Morrey BF. Results of Treatment of Fracture-Dislocations of the Elbow. Clin

[14] Orthop Relat Res. 1987 Mar ;(216):109-19. 
[15] Hudak PL, Amadio PC, Bombardier C; the Upper Extremity Collaborative Group (UECG).

[16] Development Of An Upper Extremity Outcome Measure: The DASH (Disabilities Of The Arm, Shoulder And Hand) [Corrected]. Am J Ind Med. 1996 Jun; 29(6):602-8.

[17] Brent M, Victor DS, Jasonwb, Thomas AE, Mohit B. Electrical Stimulation For Long-Bone Fracture-Healing: A Meta-Analysis Of Randomized Controlled Trials. J Bone Joint Surg Am, 2008 Nov 01; 90(11): 2322 2322-2330. Doi:10.2106/JBJS.H.00111.

[18] Chee Y, Agorastides I, Garg N, Bass A, Bruce C. Treatment Of Severely Displaced Proximal Humeral Fractures In Children With Elastic Stable Intramedullary Nailing. J Pediatr Orthop B 2006; 15(1):45-50.

[19] Odéhouri-Koudou TH, Gouli JC, Kreh JBY, Tembély S, Ouattara O, Dick KR. Elastic Stable Intramedullary Nailing In Paediatric Traumatology At Yopougon Teaching Hospital (Abidjan). Afr J Paediatr Surg 2011;8(2):155-8.

[20] Lacher M, Schaeffer K, Boehm R, Dietz HG. The Treatment Of Supracondylar Humeral Fractures With Elastic Stable Intramedullary Nailing (ESIN) In Children. J Pediatr Orthop 2011; 31(1):33-8.

[21] Xie F, Wang S, Jiao Q, Shen Y, Ying H. Minimally Invasive Treatment For Severely Displaced Proximal Humeral Fractures In Children Usingtitanium Elastic Nails. J Pediatr Orthop 2011; 31(24):839-46. 